\title{
HACIA UN ESPACIO DE INVESTIGACIÓN Y LA EDUCACIÓN SUPERIOR
}

Por: Isabel Pascual Gómez*

Carmen Lafuente Ibáñez **

\section{Resumen}

La construcción del Espacio Europeo de la Educación Superior es un proceso que se inicia con la declaración de la Sorbona en 1998, se consolida con la Declaración de Bolonia en 1999 y continuará hasta 2010 con la realización de una serie bianual de Conferencias de Ministros de Educación Superior.

Este trabajo pretende realizar una breve descripción de cada una de estas etapas del proceso de convergencia, destacar los logros, problemas y circunstancias que han encontrado las Instituciones de Educación Superior en la adaptación de sus enseñanzas al nuevo Espacio Europeo de Educación Superior (EEES), así como describir los elementos que conformarían el futuro Espacio Europeo de Investigación(EEI). Entre ellos se destaca el carácter internacional del EEl, la movilidad como medio de apoyo a la investigación y los sistemas de financiación que hace posible su realización.

\section{ALABRAS CLAVE}

Espacio Europeo de Educación Superior, convergencia, ECTS, calidad, movilidad, Espacio Europeo de Investigación, Sistema de reconocimiento de títulos, financiación y dimensión internacional.

\section{EL PROCESO DE BOLONIA}

\subsection{Declaración de la Sorbona}

El 25 de mayo de 1998 en Paris con motivo de la celebración del aniversario de la Sorbona, los ministros representantes de Francia, Alemania, Italia y Reino Unido realizan una declaración conjunta para la armonización del diseño del Sistema de Educación Superior. En esta declaración manifiestan la necesidad de promover un sistema de educación superior que permita:

- Desarrollar las dimensiones intelectuales, culturales, sociales y técnicas del continente europeo.

- Crear un área europea de educación abierta y flexible que favorezca la movilidad y cooperación entre los estudiantes, conseguida mediante el sistema de créditos ECTS (Sistema Europeo de Transferencia de Créditos).

\footnotetext{
* Isabel Pascual. Doctora en Ciencias de la Educación (UCM) y profesora de la Universidad Antonio de Nebrija.

**Carmen Lafuente. Doctora en Ciencias Económicas y Empresariales en el área de Métodos Cuantitativos y coordinadora del programa de doctorado en Ciencias Empresariales.
} 
- Facilitar a los universitarios el acceso a variedad de programas y estudios multidisciplinares, el perfeccionamiento de los idiomas y la capacidad para utilizar las nuevas tecnologías informativas.

- Establecer un sistema cíclico de enseñanza conformado por un primer nivel de grado que dará lugar a la obtención de un título con cualificación profesional en el mercado laboral europeo y un segundo nivel de posgrado, para cuyo acceso será necesario haber superado el primero y que podrá dar lugar a la obtención del Título de Master y/ o Doctorado. Ambas titulaciones de segundo ciclo, orientadas tanto a la investigación, como al trabajo autónomo.

En esta primera declaración los ministros participantes animan a otros miembros de la Unión Europea y al resto de los países europeos a participar en la creación de la Zona Europea de la Educación Superior.

\subsection{Declaración de Bolonia}

El 19 de junio de 1999 los ministros de educación reunidos en Bolonia fijan las bases para la construcción del Espacio Europeo de la Educación Superior (EEES). Al tiempo que reafirman los principios fundamentales de la Declaración de la Sorbona, se comprometen a coordinar las políticas para alcanzar a corto plazo:

- Un sistema de títulos comprensibles y comparables por medio de, entre otras medidas, el Suplemento Europeo al Título a fin de promover la empleabilidad de los ciudadanos europeos y la competitividad del sistema de enseñanza superior europeo a escala internacional.

\section{A bstract}

The construction of the European Higher Education Area is a process that began with the Declaration of the Sorbona in 1998, consolidated by the Declaration of Bologna in 1999 and will continue until 2010 with the celebration of a series of bianual conferences for Higher Education ministers.

This paper tries to sum up a brief description of each of the stages of the process of convergence, emphasizing the achievements, problems and circumstances that the Institutions of Higher Education have gone through in the improvement of their programs in the European Higher Education area, as well as a detailed description of the elements that would shape the future European research process. Among those issues we find the international character of the EEI, the mobility as a way to support research and the funding systems that make this accomplishment possible.

\section{KEY WORDS}

The European research processconvergence-ECTS- quality- mobilitysystem of certificate official approvalfinances- international dimension. 
- Un sistema de enseñanzas basado en dos ciclos principales: un primer ciclo de 3 años de duración correspondiente a un nivel de cualificación adecuado para acceder al mercado de trabajo europeo y un segundo ciclo de 1 ó 2 años de duración, que permite obtener el título de doctor.

- Un sistema de créditos ECTS reconocidos por las universidades, que permita la movilidad de los estudiantes.

- Un programa de movilidad de los estudiantes, profesores, investigadores y personal administrativo en el contexto europeo.

- Un sistema de garantía de la calidad, estableciéndose criterios comunes sobre modelos de evaluación y acreditación de estudios.

- Promocionar la dimensión europea de la educación superior elaborando programas integrados de movilidad, estudios, formación e investigación.

En esta declaración se establece, además, un compromiso público de colaboración entre las organizaciones intergubernamentales y no gubernamentales que tienen competencias en el ámbito de la Educación Superior.

\subsection{Declaración de praga}

El 19 de mayo de 2001 se reúnen 32 representantes europeos de la Educación Superior. El objetivo de esta reunión es examinar el grado de cumplimiento del Informe, Fomentando el Proceso de Bolonia. Los ministros valoran muy positivamente la incorporación al proceso de la Asociación Europea Universitaria (EUA) y las Uniones Nacionales de Estudiantes en Europa (ESIB) y realizan el siguiente balance sobre los aspectos más importantes del Proceso de Convergencia:

\section{- Sobre la estructura de ciclos}

Aunque la estructura de niveles basada en los dos ciclos principales, mencionados anteriormente, ya ha sido adoptada por algunos países y abordada y discutida en otros muchos, es necesario promover, desde las oficinas de la Red Europea de Centros de Información ( "European NetworK of Information Center" ENIC) y los Centros Nacionales de Información sobre Reconocimiento Académico ("National Academic Recognition Information Center" NARIC), un sistema de reconocimiento profesional y académico de las titulaciones, que permita a los ciudadanos usar sus cualificaciones, competencias y habilidades a lo largo del Área de Educación Superior Europea.

\section{- Sobre el sistema de créditos ECTS}

Es imprescindible adoptar el sistema de créditos ECTS o un sistema compatible a este, junto con el Suplemento al Diploma, para ofrecer una mayor flexibilidad en los sistemas de aprendizaje y calificación universitarios y facilitar el acceso de los estudiantes al mercado laboral europeo.

\section{- Sobre los programas de movilidad}

Hay que eliminar los obstáculos que impidan la libre circulación de estudiantes, profesores e investigadores tomando en consideración los Programas de la Comunidad Económica Europea y la emisión del Plan de Acción de Movilidad refrendado por el Consejo Europeo en Niza en el año 2000.

\section{- Sobre los sistemas de garantía de calidad}

Instan a las agencias estatales y a la Red Europea de Garantía de la Calidad (ENQUA) a difundir ejemplos de la mejor práctica y diseñar 
mecanismos de evaluación y acreditación/ certificación.

Adicionalmente los ministros destacan la importancia de incorporar al proceso de convergencia:

- A las instituciones universitarias y a los estudiantes en el Proceso de Convergencia.

- El concepto de formación continua o aprendizaje a lo largo de toda la vida, necesario para poder afrontar los nuevos y constantes avances tecnológicos y crear un espacio de educación competitivo.

- Programas de promoción o mejora de la educación superior europea tanto para los estudiantes europeos como para estudiantes de otras partes del mundo.

Para garantizar el éxito del Proceso de Convergencia se crea un grupo de seguimiento formado por representantes de los países firmantes, nuevos participantes como Croacia, Chipre y Turquía, la presidencia de la Unión Europea, la Comisión Europea, la Asociación Europea de Universidades, la Asociación Europea de Instituciones en Educación Superior (EURASHE), las Uniones Nacionales de Estudiantes en Europa y el Consejo de Europa.

\subsection{Berlín 2003}

El comunicado de Berlín 2003 Realizando el Espacio Europeo de Enseñanza Superior, es firmado el 19 de septiembre por los 33 ministros participantes y cuenta con la colaboración de la Asociación Europea de Universidades (EUA), la Unión Nacional de Estudiantes en Europa (ESIB), la Asociación Europea de Asociaciones Universitarias (EURASHE) y otras entidades, no todas integradas en el Proceso de Bolonia.
En éste se acuerda admitir en el Proceso de Bolonia a siete nuevos miembros:Albania, Andorra, Bosnia-Herzegoniva, Santa Sede, Rusia, Serbia y Montenegro comprometiéndose a:

- Realizar los esfuerzos que sean necesarios para asegurar la unión entre la educación superior y los sistemas de investigación fortaleciendo el área de Investigación Europea y preservando tanto la riqueza cultural europea como su diversidad lingüística.

- Reafirmar la dimensión social del proceso de Bolonia, definiendo la educación como un bien y responsabilidad públicos..

Las conclusiones del Informe de Progreso efectuado por el grupo de seguimiento, les permite puntualizar:

\section{- Sobre los sistemas de garantía de calidad}

La ENQUA, en cooperación con otras instituciones, debe desarrollar procedimientos, guías para la garantía de la calidad, así como sistemas de acreditación de agencias y cuerpos para el encuentro del 2005.

\section{- Sobre el sistema de ciclos}

Debe comenzar la implantación del sistema de dos ciclos y un marco de calificaciones comparables y compatibles en el sistema de Educación Superior, comprometiéndose a que cada estudiante que se gradúe antes del 2005 reciba gratuitamente el Suplemento europeo al Título en un amplio abanico de idiomas europeos.

\section{- Sobre la movilidad}

Deben incrementarse las figuras de movilidad facilitando el acceso a préstamos y becas. 


\section{- Sobre los créditos ECTS}

El sistema de créditos ECTS debe progresar, no sólo como sistema de transferencia, sino también como sistema de acumulación.

\section{- Sobre la promoción del EEE}

Deben identificarse nuevos caminos para incrementar la participación estudiantil en el proceso asegurándose de que todos los estudiantes pasen un periodo sustancial de estudios en el extranjero cooperando activamente con regiones de otras partes del mundo.

El nuevo grupo de seguimiento se compromete a reunirse al menos dos veces al año y lo presidirá el Estado miembro que ostente la presidencia de la UE, con la participación de los representantes de todos los miembros del proceso de Bolonia y la comisión europea, el Consejo de Europa, el EUA, EURASHE, ESIB y UNESCO/ CEPES como miembros consultivos.

\subsection{Bergen 2005}

El Informe General 2003-2005 del Grupo de Seguimiento (BFUG); el informe, Tendencias IV de la EUA y el informe de ESIB, Bolonia desde el punto de vista de los Estudiantes permiten a los ministros reunidos el 20 de mayo, por una parte, realizar un balance muy positivo sobre tres aspectos prioritarios de este proceso: la estructura de ciclos, la garantía de la calidad y el reconocimiento de títulos y periodos de estudio y, por otra, detectar las siguientes limitaciones:

\section{- Sobre el sistema de titulaciones}

Se observa que el sistema de dos ciclos se está aplicando ampliamente con algunos problemas referentes al acceso entre ellos. Por este motivo se aconseja adoptar un marco general de cualificaciones en el EEES que comprenda tres ciclos; aportar los descriptores genéricos basados en resultados del aprendizaje y competencias para cada ciclo y describir los intervalos de créditos en el primer y segundo ciclos. Este marco y el marco de cualificaciones para el aprendizaje a lo largo de toda la vida deben ser complementarios y, además, incluir una educación general y una formación profesional.

\section{- Sobre la garantía de calidad}

Se aprecia que todos los países han tomado medidas para aplicar un sistema de garantía de calidad, aunque hace falta más participación de los estudiantes y más cooperación internacional. Se adquiere el compromiso de adoptar los estándares de la ENQA e introducir el modelo de evaluación por pares de agencias de calidad nacionales, subrayando la importancia de la cooperación entre agencias reconocidas con el objeto de incrementar el reconocimiento mutuo sobre las decisiones de acreditación o garantía de la calidad.

\section{- Sobre el reconocimiento de títulos y periodos de estudio}

Se insta a los participantes que no lo han hecho, a ratificar la Convención de Reconocimiento de Lisboa (1997) y a adquirir el compromiso de diseñar planes nacionales para mejorar la calidad de los procesos asociados al reconocimiento de títulos extranjeros, teniendo en cuenta títulos conjuntos otorgados por dos o más países del EEES.

\section{- Sobre la dimensión social del proceso de convergencia}

Se renueva el compromiso para que la educación superior sea accesible para todos, estableciendo las condiciones apropiadas que 
les permita a los estudiantes completar sus estudios sin obstáculos relacionados con su origen social y económico.

\section{- Sobre la movilidad}

Se reafirma el compromiso de promover la movilidad de los estudiantes y del personal universitario, facilitando la concesión de visados y de permisos de trabajo y fomentando la participación en los programas de movilidad e intercambio con otros continentes.

Los objetivos del grupo de seguimiento para el 2007 deben centrarse en:

- La puesta en práctica de sistemas y directrices en cuanto a garantía de calidad.

- La expedición y reconocimiento de títulos conjuntos, incluido el doctorado.

- La creación de itinerarios flexibles, incluyendo procedimientos para el reconocimiento del aprendizaje previo.

- La puesta en marcha de sistemas de cualificaciones nacionales.

- El balance sobre la movilidad de los estudiantes, así como la situación social y económica de los alumnos de los países participantes.

\subsection{Londres 2007}

El 18 de mayo de este año, los ministros responsables de la Educación Superior hacen un balance de los objetivos conseguidos desde la conferencia de Bergen en el año 2005 en el comunicado: Hacia el Espacio Europeo de Educación Superior: respondiendo a los retos de un mundo globalizado.
Este informe, junto con los informes, Tendencias $V$ de la EUA (Asociación de Universidades Europeas), Bolonia desde la Perspectiva de los Estudiantes del ESIB (Consejo de Estudiantes europeos), Sobre la estructura de la Educación Superior en Europa de Eurydice, confirman los siguientes avances hacia la construcción del EEES:

\section{- Respecto a la movilidad}

Desde 1999 la movilidad, agente clave en el proceso de Bolonia, ha aumentado; sin embargo, aún existen obstáculos relacionados con la inmigración, el reconocimiento de títulos, los incentivos económicos insuficientes o disposiciones demasiado rígidas sobre la jubilación. Por lo tanto, es importante que cada gobierno se comprometa a facilitar la entrega de visados y los permisos de residencia y trabajo.

\section{- Respecto a la estructura de los estudios}

El número de estudiantes matriculados en cursos de los dos primeros ciclos ha aumentado y se han reducido las barreras estructurales entre los dos ciclos, incrementándose el número de programas de doctorado. Los esfuerzos deben centrarse en eliminar las barreras al acceso y a la progresión entre ciclos, así como en implementar adecuadamente los ECTS con base en los resultados del aprendizaje y en la carga de trabajo del estudiante.

\section{- Respecto al reconocimiento}

Se ha avanzado en la puesta en marcha de la Convención de Lisboa sobre reconocimiento en los créditos ECTS, pero es necesario mayor uniformidad y coherencia. 


\section{- Respecto a las cualificaciones}

Sigue siendo necesario poner en práctica los marcos nacionales de cualificaciones acreditados por el Modelo Global del Marco de Cualifiaciones del EEES.

\section{- Sobre el aprendizaje a lo largo de toda la vida}

En la mayor parte de los países existen elementos de aprendizaje flexible, pero es necesario el desarrollo de itinerarios de aprendizaje desde etapas más tempranas, previas a la Educación Superior.

\section{- Sobre la Certificación de la Calidad}

La certificación externa de Calidad está mucho más desarrollada, por lo que el número de estudiantes se ha venido incrementando desde el año 2005. Se subraya la necesidad de crear un Registro Europeo de Agencias Certificadoras de Calidad de la Enseñanza Superior de carácter voluntario, autosuficiente económicamente, independiente y transparente.

\section{- Sobre la dimensión social}

Se continúan haciendo esfuerzos para brindar a los estudiantes servicios adecuados y crear itinerarios de aprendizaje más flexibles, tanto para acceder a la educación superior, como, una vez dentro, ampliar la participación a todo nivel, sobre la base de la igualdad de oportunidades.

\section{- Sobre el Área Europea de Investigación}

El acercamiento entre el EEES y el EEI es un objetivo importante por lo que se han desarrollado y mantenido una amplia variedad de programas de doctorado ligados al modelo global del marco de cualificaciones del EEES. Sin embargo, sigue siendo necesario mejorar el estatus, las perspectivas profesionales y la financiación de los investigadores que inician sus carreras.

El grupo de seguimiento, de cara a los próximos dos años, acuerda culminar las líneas de acción aprobadas y obtener:

- Informes nacionales de cada país donde se destaquen las medidas tomadas para promover la movilidad de los estudiantes y el personal, así como las acciones para evaluarlas en el futuro; los datos sobre la movilidad en todos los países participantes en el Proceso de Bolonia; las estrategias y políticas nacionales para la promoción de la dimensión social y las acciones que se deben adoptar para incrementar la empleabilidad y el aprendizaje a lo largo de toda la vida.

- Un informe del Grupo de Seguimiento de Bolonia en 2009 que muestre los progresos globales en Europa, tanto en lo estatal como en lo institucional. 


\section{Puesta en PRÁCtica de las Reformas de BOLONIA POR PARTE DE LAS UNIVERSIDADES}

\begin{abstract}
Tendencias IV: Universidades europeas. Puesta en práctica de Bolonia es un informe de naturaleza cualitativa elaborado por un grupo de expertos universitarios de la "European University Association", tras realizar visitas y entrevistas a sesentay dos universidades euro-peas; constituye una descripción exhaustiva y actualizada del grado de aplicación de las reformas.
\end{abstract}

El grupo de expertos refleja en este informe que con un amplio consenso las universidades han aceptado los retos que plantea la puesta en práctica de Bolonia. Sin embargo, también han encontrado en las instituciones de Enseñanza Superior reticencias de carácter global en aspectos como la insuficiente autonomía universitaria institucional o la falta de recursos económicos necesarios para hacer frente a la reestructuración y, de carácter particular, en aspectos concretos derivados de la puesta en práctica del Proceso de Convergencia.

A continuación vamos a sintetizar, siguiendo la misma estructura que en el apartado anterior, algunos de los inconvenientes o problemas reseñados por las instituciones entrevistadas.

\section{- Sobre la estructura de las titulaciones}

La revisión de los planes de estudios en los grados está en fase de implantación pero no así su racionalización. Existe la impresión de que hay que enseñar las mismas asignaturas en menos tiempo para cumplir los requisitos legales vigentes, por lo que se corre el riesgo de que se implanten conceptos y herramientas tales como la enseñanza centrada en el aprendizaje, los resultados de aprendizaje, la modularización de los planes y la relación de los créditos ECTS y el suplemento al Diploma, sin comprender realmente sus funciones pedagógicas.

Las titulaciones de grado carecen de credibilidad entre algunos profesores, estudiantes y empresarios. Los gobiernos de los distintos países deberían trabajar conjuntamente con las Cámaras de Comercio estableciendo convenios de colaboración de fomento tanto del empleo público como privado de sus titulados de grado.

La cuestión de la duración de los estudios en posgrado y la distinción entre los masters profesionales y académicos sigue siendo polémica en algunos países. Estos países creen que los estudios de grado y de posgrado deberían concebirse como partes de un todo.

La posibilidad de expedir titulaciones conjuntas está progresando positivamente, aunque uno de los mayores problemas de carácter práctico que se plantean es el que tiene que ver con la evaluación de la calidad y la acreditación.

\section{- Sobre el reconocimiento}

El uso del ECTS está muy implantado pero existen problemas a la hora de asignar créditos a las asignaturas, especialmente cuando se tiene que valorar correctamente la carga de trabajo del estudiante.

El reconocimiento de la movilidad se ve muy favorecido por la creación de convenios de intercambio, pero no siempre es posible un reconocimiento total. Es necesario adoptar calendarios académicos comunes con acuerdos sobre la fecha de finalización de los semestres. Respecto al uso del Suplemento al Diploma hay que solucionar dos problemas: uno de 
carácter técnico, la necesidad de diseñar aplicaciones informáticas que faciliten el flujo de información entre las facultades y la administración central y otro terminológico, la necesidad de aplicar normativa común .

Los estudios no formales y la experiencia práctica previa deben ser reconocidos por las IES, así como los títulos extranjeros que, en muchas ocasiones, se producen a través de lentos y engorrosos procedimientos administrativos.

Los ministerios de los distintos países deberían, por lo tanto, renunciar a sus competencias en esta materia y transferirlas a las instituciones de enseñanza superior, estableciendo una estrecha colaboración entre éstas y ENIC y NARIC, organismos encargados de aplicar los principios de la Convención de Lisboa.

\section{- Sobre los sistemas de calidad}

Se observa que los procesos internos de calidad han crecido considerablemente en todas las instituciones de enseñanza superior.
Son procedimientos muy variados en su forma y contenido, relacionados especialmente con la evaluación de la docencia y el aprendizaje, pues son muy pocas las instituciones que hacen un seguimiento de la información básica en cuanto a los índices de éxito y abandono de sus estudiantes.

No existen prácticamente evaluaciones de los departamentos de administración y servicios.

Respecto a la evaluación externa, ésta suele considerarse como una carga burocrática de escasa utilidad para el desarrollo institucional, orientada más a la comprobación del cumplimiento de normas, que a conocer las prioridades, objetivos y circunstancias particulares de las instituciones evaluadas.

Por el papel esencial que ocupa el Espacio Europeo de Investigación en la Educación Superior de la Europa del conocimiento se desarrolla el tema con mayor profundidad en el epígrafe siguiente, con base en las distintas comunicaciones de la Comisión Europea.

\section{ESPACIO EUROPEO DE INVESTIGACIÓN. (EEI)}

\subsection{Contexto}

Europa se enfrenta al reto de construir una Sociedad del Conocimiento, una sociedad en la que la educación, la formación, la investigación y la tecnología deben jugar un papel fundamental, teniendo en cuenta los, ya existentes y en continuo progreso, sistemas de información.

El desarrollo de una sociedad depende de la cualificación de sus agentes económicos y sociales. La investigación y la tecnología deben ser los motores que permitan generar el conocimiento necesario para el crecimiento económico y social de cualquier economía.

En este sentido, para que Europa mantenga y aumente su competitividad y crecimiento debe apostar por la investigación y la tecnología. Sólo a través de una Sociedad del Conocimiento, podrá seguir siendo una de las principales potencias en la aportación mundial de conocimiento científico. 
Sin embargo, la situación actual no es la más idónea para conseguir este objetivo. En Europa sólo el $1,8 \%$ del PIB se destina a investigación, frente a casi un $3 \%$ de EEUU y Japón; el número de investigadores por cada mil empleados es aproximadamente el doble en estos dos países. Europa sigue aumentando las importaciones en tecnología, mientras que un alto porcentaje de europeos desarrolla sus doctorados en EEUU y se queda allí para seguir investigando, debido a la escasa integración del espacio científico y tecnológico de sus países. Cada país de la UE tiene sus propias políticas y programas de investigación y tecnología lo que redunda en una menor inversión total en el conocimiento (COM 2000, 6 final 18.1.2000).

A esta situación hay que añadir el envejecimiento de la pirámide demográfica de la UE y su negativa repercursion en la investigación, debido a la jubilación de generaciones de más edad (COM 2007, 161 final 4.4.2007).

En este contexto, para construir una Europa del Conocimiento, es necesario crear un Espacio Europeo de Investigación Superior (EEI) . Esta propuesta fue realizada por la Comisión Europea al Consejo, al Parlamento Europeo, al Comité Económico y Social y al Comité de las Regiones en la comunicación de 2000 (COM 2000, 6 final 18.1.2000). En términos de esa misma comunicación es necesario “...definir un planteamiento político que permita dinamizar de nuevo la investigación en Europa"

\subsection{Características y concepto del EEI}

En las distintas comunicaciones realizadas por la Comisión de las Comunidades Europeas en el periodo 2000-2007, el EEI se considera la piedra angular de una sociedad europea del conocimiento.
Según la Comisión, el concepto de EEI debe combinar los siguientes elementos: un mercado interior europeo de la investigación en el que los investigadores, la tecnología y los conocimientos circulen libremente; la coordinación efectiva de las actividades, los programas y políticas de investigación nacionales y regionales y un sistema de financiación y ejecución de los proyectos en el ámbito europeo.

La Comisión propone crear un mercado de investigación que permita superar las debilidades de la investigación en Europa: una financiación insuficiente, un entorno que no estimula la investigación, ni la explotación de sus resultados, la fragmentación de las actividades y la dispersión de los recursos.

Para fortalecer la investigación, el Espacio Europeo de Investigación debe reunir las siguientes características o elementos (COM 20006 final 18.1.2000):

- Infraestructuras de investigación de categoría mundial.

- Recursos humanos más abundantes y móviles.

- Instituciones de investigación excelentes.

- Puesta en común efectiva de los conocimientos.

- Programas y prioridades de investigación bien coordinados.

- Apertura del Espacio Europeo de Investigación al mundo.

\subsection{Movilidad. un instrumento de apoyo a la investigación}

La comunicación de octubre de 2000 (COM 2000, 612 final, 4.10.2000) propone orientaciones para las acciones de la Unión en el ámbito de la investigación. Estas orientaciones están dirigidas a mejorar los resultados de la investigación, a apoyarla en y para las PYME, a reforzar las 
infraestructuras, a ayudar a los investigadores y a establecer un nuevo contrato entre ciencia y sociedad.

Una sociedad basada en el conocimiento necesita un número suficiente de recursos humanos cualificados para investigar. Existen razones de peso, como la escasa mano de obra en la investigación, la "fuga de cerebros", la demografía o la poca participación de la mujer, entre otras, para afirmar que Europa necesita investigadores cualificados.

En este sentido, la movilidad se considera un instrumento eficaz para formar personas capacitadas y difundir los conocimientos ya que permite crear equipos y redes de investigación procedentes de distintos países. Asimismo aumenta la cantidad y la calidad de la formación, fomenta la cooperación internacional y favorece el intercambio de conocimientos; por lo tanto, es una herramienta imprescindible para alcanzar los objetivos del Espacio Europeo de Investigación.

La Comisión, en la comunicación de junio de 2001 (COM 2001, 331 final, 20.06.2001), presenta una estrategia dirigida a crear un entorno favorable para la movilidad de los investigadores en el EEI.

Esta estrategia se propone conseguir una Europa atractiva para los investigadores y un estímulo a la movilidad transnacional y multisectorial entre universidad e industria.

La misma comunicación propone las siguientes acciones para la aplicación de la estrategia de apoyo a la movilidad:

- Mejorar la información de la movilidad a través de una mejor publicación de vacantes y de mejores estadísticas de los estudios sobre movilidad de los investigadores.
- Mejorar la ayuda práctica a los investigadores creando centros de movilidad y métodos de contratación más ágiles.

- Fomentar la colaboración entre los estados miembros para elaborar y comparar estrategias de movilidad y para intercambiar prácticas de interés común.

- Mejorar la situación jurídica en temas de visados de entrada, de acceso al empleo y de seguridad social e imposición.

- Establecer un sistema de financiación que no se limite a la concesión de becas y que permita por un lado, la vuelta al país de origen y la reintegración en Europa, y por otro, un sistema que considere la situación de las familias y la compensación financiera concedida a cambio de las dificultades derivadas de la movilidad.

Pero no sólo deben llevarse a cabo acciones de apoyo a la movilidad para favorecer la generación de conocimiento científico y tecnológico;,además, deben proponerse medidas dirigidas a aumentar el número de investigadores. Algunas de estas iniciativas las propone la Comisión en la comunicación de octubre de 2000 (COM 2000, 612 final de 4.10.2000):

- Estimular el desarrollo del empleo científico y técnico en Europa.

- Favorecer la presencia de las mujeres en el ámbito científico.

- Alentar a los jóvenes a iniciar carreras científicas y a participar en la actividad de investigación. 


\subsection{Dimensión internacional del espacio europeo de investiga- ción estrategia de cooperación}

En la comunicación de junio de 2001 (COM 2001, 346 FINAL 25.06.2001) se recogen las sugerencias y orientaciones para crear un EEI con apertura mundial.

El principal objetivo de esta comunicación es establecer las directrices de una nueva estrategia de cooperación científica y tecnológica internacional tomando como base las enseñanzas del pasado para dotar de una dimensión internacional al EEI.

La apertura al mundo del Espacio Europeo de Investigación exige:

- Un espíritu de acción y coordinación entre todos los países de Europa.

- Establecer asociaciones científicas con los países en desarrollo para aumentar su capacidad de investigación e innovación tecnológica.

- Reforzar la cooperación con los países industrializados y los de economía emergente para compartir conocimientos y competencias.

- Reforzar la presencia de la UE en las acciones iniciadas por las organizaciones internacionales.

Para dotar de una dimensión internacional al EEI se debe establecer una estrategia cuyos mecanismos atraigan a los mejores científicos, faciliten conocimientos y tecnología del resto del mundo a los investigadores europeos, dirigan la ciencia y la tecnología a la generación de conocimiento y, por ende, permitan resolver los problemas de la seguridad mundial (alimentaria, ambiental y sanitaria).
En este sentido, debe desarrollarse una política clara y estructurada, dotada de los instrumentos necesarios que promuevan el diálogo, la movilidad de los científicos y la cooperación científica y tecnológica para un desarrollo socioeconómico duradero.

Esta política de cooperación científica y tecnológica internacional debe concretarse en el desarrollo de las siguientes actividades:

- Mejorar las condiciones de acogida de los investigadores en el EEl.

- Financiar la movilidad de los investigadores.

- Abrirse a la participación de investigadores y organizaciones de terceros países.

- Desarrollar actividades de investigación diferenciada según la problemática de de cada área, zona o región.

- Ampliar la observación tecnológica internacional (unificar y analizar tendencias científicas, tecnológias y de mercados; detectar oportunidades de cooperación y reforzar la transmisión de la información a los interesados).

- Asociar las políticas de exterior y de ayuda al desarrollo con la política de cooperación científica de la Unión.

- Cooperar con organizaciones internacionales para dar respuesta a problemas de dimensión mundial.

\subsection{Financiación}

En las diferentes comunicaciones de la Comisión ha quedado patente la inferioridad de los recursos de la UE destinados a inversiones en $\mathrm{I}+\mathrm{D}+\mathrm{i}$ respecto a países como EEUU y Japón. Del análisis realizado por esta en la comunicación de septiembre de 2002 (COM 2002,499 final 11.9.2002) se confirma la necesidad de mejorar la eficacia del sistema europeo de I+D+i y la necesidad de aumentar las inversiones en investigación y desarrollo. 
Para solucionar la escasez de inversión, los estados miembros acordaron en el año 2000 el cumplimiento de un doble objetivo para 2010:

- Aumentar la inversiones en in I+D hasta acercarlas al 3\% del PIB por término medio.

- Aumentar el porcentaje de financiación de las empresas hasta el $75 \%$ del total de las inversiones en I+D.

El cumplimiento del primer objetivo no es tarea fácil para todos los estados miembros actuales o futuros. Algunos países ya han alcanzado el objetivo, mientras que otros no lo conseguirán. Será necesario, en este último caso apelar al esfuerzo de todos para conseguir que toda la UE se beneficie de este aumento en la inversión.

El cumplimiento del segundo objetivo requerirá del establecimiento tanto de políticas que transformen las mayores inversiones en nuevos productos y servicios, como de políticas que aumenten el crecimiento de la competitividad y el empleo. Es decir, políticas dirigidas a convencer a las empresas de los beneficios derivados de la inversión en investigación y desarrollo.

En general, el aumento de las inversiones en I+D depende de unas condiciones marco favorables ya desarrolladas en comunicaciones anteriores, tales como la existencia de recursos humanos y una investigación pública de base. Para conseguir los objetivos propuestos se requiere de más investigadores y más cualificados. Esta es una condición esencial para invertir. Invertir y aumentar los recursos humanos exige el desarrollo conjunto de acciones, ya que para alcanzar un objetivo es necesario la consecución del otro y viceversa.

Además de esta condición, es importante la presencia de mercados financieros que apoyen en las diversas fases del desarrollo de empresas de alta tecnología y de otras empresas innovadoras. Los bonos y préstamos del Banco Europeo de Inversiones (BEI) pueden convertirse en una importante fuente de financiación para empresas de tamaño medio.

También son esenciales otras condiciones, entre ellas, sistemas adecuados de derecho de propiedad intelectual, iniciativa empresarial y reglamentación favorecedora de la investigación y la innovación.

Aun, existiendo condiciones favorables, debe realizarse un uso más eficaz de la financiación pública para la I+D de las empresas. Las autoridades públicas disponen de una gama de instrumentos financieros de apoyo a la inversión privada. Entre estas medidas se encuentran el apoyo directo, los incentivos fiscales, los sistemas de garantía y el apoyo público al capital riesgo.

El apoyo directo debería utilizarse en aquellas áreas donde el sector privado encuentra obstáculos importantes para invertir. Como instrumentos se pueden utilizar los subsidios, ayudas a la competencia, licitaciones y subvenciones reembolsables.

Los incentivos fiscales son cada vez más utilizados por diferentes países; los créditos fiscales están volviéndose más populares que las exenciones fiscales.

Los mecanismos de garantía variarán según el tipo de empresa de que se trate. En el caso de nuevas empresas de alta tecnología, las garantías de capital pueden estimular la inversión reduciendo el nivel de riesgo y aumentando el índice de rendimiento. Las garantías de préstamos suelen ser más adecuadas para las PYMES de los sectores tradicionales, debido a que estas prefieren la financiación mediante deuda. 
El capital riesgo es un instrumento que todavía no es muy utilizado en Europa, pero que puede resultar beneficioso para financiar las primeras fases de creación de una empresa.

Por último, cabe resaltar la puesta en marcha, desde la década de los 80 , de los diferentes Programas Marcos de la Investigación y el Desarrollo Tecnológico. Los Programas Marco son el principal instrumento de la UE en materia de financiación de la investigación en Europa.
La Comisión Europea en su comunicación de 6 de abril de 2005 (COM 2005, 119 final 6.4.2005) propone la puesta en marcha del Séptimo Programa Marco para el periodo 2007-2013, y así contribuir de forma sustancial en el objetivo de hacer de Europa una economía basada en el conocimiento.

El presupuesto para los próximos siete años es de algo más de 50.000 millones de euros distribuidos entre los cuatro bloques principales de actividades que conforman cuatro programas específicos, más un quinto programa sobre investigación nuclear.

Figura 1. Desglose indicativo del Séptimo programa marco

\begin{tabular}{|c|c|}
\hline $\begin{array}{l}\text { COOPERACIÓN } \\
\text { (64\% del presupuesto) }\end{array}$ & 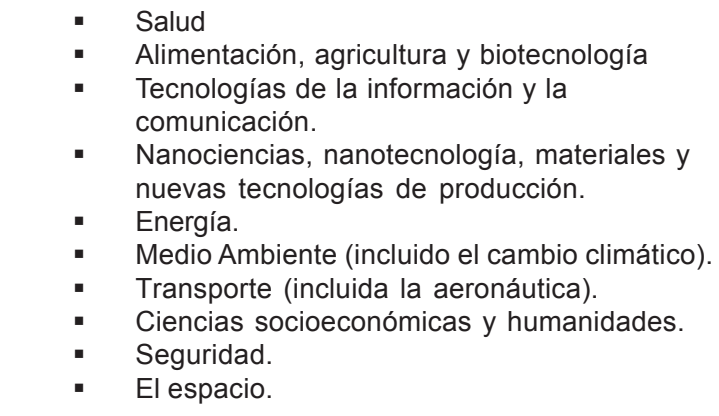 \\
\hline $\begin{array}{l}\text { IDEAS } \\
\text { (15\% del presupuesto) }\end{array}$ & $\begin{array}{l}\text { Acciones de investigación en las fronteras del } \\
\text { conocimiento. }\end{array}$ \\
\hline $\begin{array}{l}\text { PERSONAS } \\
\text { ( } 9 \% \text { del presupuesto) }\end{array}$ & $\begin{array}{l}\text { - Formación inicial de investigadores-Redes } \\
\text { Marie Curie. } \\
\text { - Formación permanente y promoción } \\
\text { profesional-becas individuales. } \\
\text { Pasarelas y asociaciones entre la industria y } \\
\text { la universidad. } \\
\text { La dimensión internacional-becas de salida a } \\
\text { terceros países, régimen de cooperación, } \\
\text { subvenciones de reincorporación. } \\
\text { - Premios a la excelencia. }\end{array}$ \\
\hline $\begin{array}{l}\text { CAPACIDADES } \\
\text { (8\% del presupuesto) }\end{array}$ & $\begin{array}{l}\text { - Infraestructuras de investigación. } \\
\text { - } \quad \text { Regiones del conocimiento. } \\
\text { - } \quad \text { Potencial de investigación. } \\
\text { - } \quad \text { Aponcia y Sociedad. } \\
\text { investigación. } \\
\text { - Actividades específicas de cooperación } \\
\text { internacional. }\end{array}$ \\
\hline $\begin{array}{l}\text { EURATOM } \\
\text { (6\% del presupuesto) }\end{array}$ & - Investigación nuclear y formación. \\
\hline
\end{tabular}

Fuente: Elaboración propia a partir de 7PM Las respuestas del mañana empiezan hoy mismo. Comisión Europea. www.ec.europa.eu/research. 
Según se desprende de la figura 1, el 7PM contempla cinco programas:

- El programa de Cooperación recoge las 10 áreas temáticas de investigación de cooperación internacional (dentro y fuera de la UE) a las que se destinará aproximadamente el $64 \%$ del presupuesto del 7PM.

- Otro 15\% irá dirigido al programa Ideas. Este se pondrá en práctica por medio del Consejo Europeo de Investigación (CEI) para impulsar la competitividad de Europa a través de la financiación de la investigación de alta calidad en todos los campos de la ciencia.
- La formación, la oportunidad de poder desarrollar una carrera en la investigación y la movilidad están dentro del programa Personas, que absorbe el $9 \%$ del presupuesto.

- El $8 \%$ del prepuesto se destinará a incrementar y optimizar las infraestructuras de investigación.

- El 6\% restante corresponde al programa Euratom cuyo objetivo es fomentar la investigación y formación en materia nuclear.

\section{Conclusiones}

Desde la primera Declaración de la Sorbona en 1998, ha habido un largo proceso de declaraciones de los Estados miembros de la UE y de comunicaciones de la Comisión Europea en las que se ha reconocido, sin reservas, por una parte, la importancia de una educación de alta calidad y de una excelencia investigadora para construir una Europa del Conocimiento que garantice el desarrollo económico y social y, por otra,un mismo objetivo y una realidad con grandes diferencias culturales, sociales, económicas y morales.

La construcción del sistema que engloba al EEES y al EEI debe hacerse respetando la diversidad del marco social existente, con la colaboración de instituciones fuertes y responsables adecuadamente financiadas y con capacidad de financiar. Las política públicas, las empresas, las universidades, los estudiantes... deben dirigir sus esfuerzos hacia un sistema común.

En todo este periodo se han logrado algunos avances para conseguir una estructura más homogénea en titulaciones de grado y posgrado, se han creado plataformas tecnológicas para una mejor coordinación de las actividades y los programas de investigación, ha aumentado la financiación de la UE a través del Séptimo Programa Marco, las empresas destinan un 5\% más en I+D, ...y, aunque todavía queda mucho por hacer, el sistema europeo de educación superior e investigación continúa desarrollándose. 
- Declaración de Bolonia (1999). Joint Declaration of the European Ministers of Education.

- COM (2000) 6 final de 18.1.2000. Comunicación de la Comisión al Consejo, al Parlamento Europeo, al Comité Económico y Social y al Comité de las regiones. Hacia un Espacio Europeo de Investigación.

- $\operatorname{COM}(2000) 612$ final de 4.10.2000. Comunicación de la Comisión al Consejo, al Parlamento Europeo, al Comité Económico y Social y al Comité de las regiones. Realización del Espacio Europeo de Investigación: orientaciones par a las acciones de la Unión en el ámbito de la investigación (2002-2006).

- COM (2001) 331 final de 20.6.2001. Comunicación de la Comisión al Consejo y al Parlamento Europeo. Una estrategia de movilidad para el Espacio Europeo de Investigación.

- COM (2001) 346 final de 25.6.2001. Comunicación de la Comisión. La dimension internacional del Espacio Europeo de Investigación.

- COM (2001) 549 final de 3.10.2001. Comunicación de la Comisión. La dimension regional del Espacio Europeo de Investigación.

- COM (2002) 499 final de11.9.2002. Comunicación de la Comisión. Más investigación para Europa:3\% del PIB.

- COM (2003) 436 final de 18.7.2003. Comunicación de la Comisión al Consejo y al Parlamento Europeo. Los investigadores en el espacio europeo de la investigación: Una profesión con multiples carreras.

- COM (2004) 353 final de 16.6.2004. Comunicación de la Comisión. La ciencia y la tecnología, claves del futuro de europa-Orientaciones para la política de apoyo a la investigación de la Unión.

- COM (2005) 118 final de 6.4.2005. Comunicación de la Comisión. Construcción del Espacio Europeo de la Investigación al servicio del crecimiento.

- COM (2007) 161 final de 4.4.2007. Libro verde. El Espacio Europeo de Investigación: nuevas perspectivas.

- Comunicado de Praga (2001). Meeting of European Ministers in charge of Higher Education.

- Comunicado de Berlin (2003). Conference of Ministers responsible for Higher Education.

- Comunicado de Bergen (2005). Conference of Ministers responsible for Higher Education.

- Comunicado de Londres (2007). Conference of Ministers responsible for Higher Education.

- Guía del Espacio Europeo de la Educación Superior (2006). Red de Información Europea de la Comunidad de Madrid.

- Reichert, S., Tauch C. (2005). Tendencias IV : Universidades Europeas. Puesta en práctica de Bolonia. Informe Técnico de la European University Association. 